\title{
Extended testing for cognition: has awake brain mapping moved to the next level?
}

\author{
George Samandouras ${ }^{1}$ (1)
}

Received: 27 September 2021 / Accepted: 28 September 2021 / Published online: 10 November 2021

(c) The Author(s), under exclusive licence to Springer-Verlag GmbH Austria, part of Springer Nature 2021

\section{Evolution of mapping concepts}

Penfield, as depicted masterfully in the 1936 Boldrey's thesis, produced a detailed, direct electrical stimulation (DES)generated, motor, somatotopic map, including specific stimulation sites of individual fingers [17, 21]. Harvey Cushing, Penfield's remote mentor, had stimulated non-human primates while working in England with renowned neurophysiologist, and later, Nobel laureate, Charles Sherrington, and produced in 1902 and 1903 rather coarse human maps with joint movements only [21]. Some 50 years earlier, in 1884 , Victor Horsley had performed the first successfully organized intraoperative stimulation of a human cerebral cortex in a case of occipital encephalocele resulting in rapid conjugate eye deviation, attributed by Horsley to the stimulation of the quadrigeminal plate [36].

Penfield is also credited for mapping language, modifying Foerster's technique, and applied DES with bipolar electrodes producing a square waves with a frequency of $60 \mathrm{~Hz}$ and a duration of 0.2 to $0.5 \mathrm{~ms}$, not remote to current settings $[17,30]$. Applying similar principles, Ojemann, in a landmark 1983 paper, produced a probabilistic model of language organization derived from stimulation mapping of language-related functions including naming, reading, shortterm verbal memory, mimicry of orofacial movements, and phoneme identification, thereby setting the foundations of modern intraoperative language mapping [26].

During the 4 subsequent decades, awake brain mapping $(\mathrm{ABM})$ evolved exponentially, as accumulated literature suggested that, at least in glioma surgery, ABM maximised safe resections, representing a standard of care $[9,40]$. Indeed, in the largest meta-analysis totalling 8,091 adult patients who underwent tumour surgery with and without $A B M$,

George Samandouras

g.samandouras@ucl.ac.uk

1 The National Hospital for Neurology and Neurosurgery, Queen Square, London, UK gross total resections were documented in $75 \%$ and $58 \%$ of patients, respectively, while late, severe neurological deficits were observed in $3.4 \%$ and $8.2 \%$ of patients, respectively [9]. However, as accrued data were analysed, a number of cognitive impairments appeared to emerge either as a result of neoplastic invasion or post-surgery sequelae, prompting the development of intraoperative tasks to test, detect, and protect, higher cognitive functions beyond language and movement, during ABM [4, 25, 33]. In a thoughtful meta-analysis, Ruis confirmed, quite convincingly, this gap in intraoperative testing, showing that among 232 qualifying studies, testing of memory, emotions, calculations, or other cognitive functions beyond language was performed or reported extremely rarely [31].

\section{Current systematic review}

In this issue of Acta, Landers et al. focused their efforts in systematically reviewing published evidence on the use of DES in white matter tracts subserving visuospatial attention and a group of three executive functions including inhibitory control, working memory, and cognitive flexibility [20]. Conclusions drawn from eleven qualifying studies indicated that the right SLF-II is involved in visuospatial awareness, as previously and rather extensively has already shown, while the left SLF-III and possibly the right SLF-I are involved in working memory; the cingulum appeared to be implicated in cognitive flexibility [20]. The authors insightfully recognised that their conclusions, based on the heterogeneity of study types and employed methods, could not unequivocally support their emerging hypotheses.

Beyond the unusual grouping of visuospatial attention with three core executive functions, with the former dominating the study populations with seven out of eleven studies, Landers et al. should be commended for investigating and drawing attention to intraoperative mapping of top-tier cognitive domains. As neurosurgeons interrelate 
increasingly with cognitive neuroscience, a working knowledge of what constitutes cognitive domains, and in this particular paper, what represents executive functions, may be useful, as definitions in the literature are far from consistent. Cognitive domains are numerous, distinct, hierarchical, and interrelated with executive functioning and cognitive control occupying the top hierarchy tier [15]. The cognitive subdomains of executive functions, in particular, involve reasoning and problem solving, in other words, "real-world adaptive success" [15, 32].

In addition, memory mapping has received limited attention in the literature although neurology studies have shown to be severely impaired in diverse anatomical substrates including cortical dementias and frontostriatal disorders and a variety of neuropsychiatric disorders affecting subdomains such as procedural, affective, prospective, and manipulation working memory $[14,15]$. With regard to working memory as a core executive function, beyond operational definitions, it should be noted that the current systematic review could not power any conclusions. For example, the involvement of right SLF-I in working memory was based in one study of two patients. Therefore, in this specific white matter tract, caution is advised when extrapolating a single report of two cases as results of a systematic review.

A second challenge when interpreting DES outcome reports is the reliance on the operators' interpretation during surgery. For example, since Makris introduced in 2004 the anatomical definition of SLF I, its anatomical existence has been disputed in a number of studies as was found in minority of subjects and attributed to be part of the cingulum in all cases of one study, as confirmed on both DSI and HCP templates [23, 37]. Although our group has consistently identified in white matter dissections the wavy, SLF I, in the upper bank of the cingulate sulcus, connecting the precuneus with the medial superior frontal gyrus, this controversy demonstrates how challenging it does remain to dissect, among other tracts, SLF I, even in an ideal, laboratory environment $[37,38]$. Therefore reports on its reliable intraoperative identification and stimulation, particularly in a novel relation to working memory, should be considered carefully.

It remains always contingent on the surgeons/team, experience, and expertise to know what exactly the surgeon is stimulating, what is the proposed function of the stimulated region, what is the appropriate test for the proposed function, and how any results can be thresholded and interpreted, in the complex surgical environment. For example, although working memory impairment was attributed to SLF I in the cited paper, a more sustained attention compromise, subserved by medial frontal regions, may have contaminated working memory assessment [35].

\section{Brain mapping challenges}

These methodological challenges lead to a number of observations, often overlooked, in ABM studies, which are closely linked to wider neuroscience issues. First, successful ABM outcomes are predicated on clear understanding of the anatomical definitions, presumed function, and appropriate, intraoperative testing paradigms. However, a strictly defined, consensual map of cortical parcels and subcortical white mater segments is not currently available. Since Brodmann's 43 cytoarchitectonic areas, Vogt's and Vogt's 185 myeloarchitectonic areas, and von Economo and Koskinas' 107 regions, multiple parcellation maps have been generated, mostly clinically unvalidated [1, 2, 13, 24].

Notwithstanding the undisputed presence of neural networks and metanetworks, neurosurgeons need accurate anatomical definitions of areas such as the dorsolateral prefrontal cortex or ventral or dorsal premotor cortices, among others, so region-specific DES can be applied. Whether these specialised cortical parcels represent hyperconnected network hubs, regions of high nodal connectedness, or cortical epicentres, should not distract form the need of their accurate topological definition [8]. Many excellent probabilistic maps have been recently proposed, but these cannot be applied anatomically, without significant interobserver variability [28, 32]. Similarly, regardless if the brain function frameworks are topological or, most likely, hodotopical, as initially supported more than a century and a half ago, and regardless of the re-organisational plasticity of the neural tissue, certain network disruptions can and will inflict neurological deficits, in motor, language, and other cognitive domains $[5-8,19]$.

Second, the precise function of most cortical parcels and subcortical white mater tracts remains incomplete. For example, the arcuate fasciculus, perhaps the most widely studied language pathway, has been represented with conflicting anatomo-functional models as proposed sequentially by De'jerine in 1895, Geschwind in 1970, Catani in 2005, and Glasser and Rilling in 2008 and numerous other tractographic, track-tracing, and fibre dissection models $[6,10$, $12,13,38]$.

Third, there is currently significant interobserver variability in selection and interpretation of intraoperative testing for language and other cognitive domains regardless of specialism or years of experience [34]. Our group documented lack of consensus among experienced specialists in interpreting common terminology (for example, definitions such as anomia or apraxia of speech) or selection of intraoperative tests (such as selecting tasks for the dominant dorsolateral prefrontal cortex or dominant parts triangularis) [34]. 


\section{Mapping cognition}

These methodological limitations, lack of standardization, and often lack of agreement on what is tested and how, even when testing structures we have been electrically stimulating for many decades, should be considered when wider cognitive functions beyond language are interrogated. Fortunately, a wealth of initial DES data is emerging from high-quality cognitive studies [22, 27, 32]. Recent reports accrue data on specific testing of wider cognitive domains including cognitive aspects of movement, such as awareness of voluntary actions using the hand manipulation task; interference control or achieving a goal through conflicting incoming information using the Stroop test; and face-based mentalising or mental state recognition using the "reading the mind in the eyes" test $[3,11,29,39]$. It should be noted however that highly distributed rather than modular, context-sensitive cognitive metanetworks involving emotion, spatial cognition, and memory, among others, would be significantly more challenging to map [16].

ABM remains a cornerstone not only of maximum safe resection of gliomas, prolonging survival and protecting patients' quality of life, but also validating emerging cognitive theories and generating novel, invaluable data in the functional organisation of movement, language, and other cognitive domains. It should be emphasized, however, that certain high order, flexible cognitive functions are subserved by widespread, large-scale, interacting, synchronised networks and a brief, temporary DES disconnection may not access specific cognitive subdomains or indeed may disconnect interacting networks; therefore, excessive claims should be tempered [16]. It is hoped that by reducing methodological and interobserver variabilities, producing precise and validated anatomo-functional maps, standardising mapping paradigms with inclusion of personalised data, and possibly creating inter-institutional registries of DES outputs, ABM will protect further patients' quality of life and strengthen its unique contributions to cognitive and wider neuroscience [18, 32, 34].

\section{References}

1. Amunts K, Lepage C, Borgeat L et al (2013) BigBrain: An ultrahigh-resolution 3D human brain model. Science 340(6139):1472-1475

2. Amunts K, Zilles K (2015) Architectonic mapping of the human brain beyond Brodmann. Neuron 88(6):1086-1107

3. Baron-Cohen S, Wheelwright S, Skinner R, Martin J, Clubley E (2001) The autism-spectrum quotient (AQ): evidence from Asperger syndrome/high-functioning autism, males and females, scientists and mathematicians. J Autism Dev Disord 31(1):5-17
4. Barzilai O, Ben Moshe S, Sitt R, Sela G, Shofty B, Ram Z (2018) Improvement in cognitive function after surgery for lowgrade glioma. J Neurosurg 130(2):426-434

5. Catani M, Ffytche DH (2005) The rises and falls of disconnection syndromes. Brain 128(10):2224-2239

6. Catani M, Jones DK, Ffytche DH (2005) Perisylvian language networks of the human brain. Annals of neurology 57(1):8-16

7. Cramer SC (2008) Repairing the human brain after stroke: I Mechanisms of spontaneous recovery. Annals of neurology 63(3):272-287

8. Dadario NB, Brahimaj B, Yeung J, Sughrue ME (2021) Reducing the cognitive footprint of brain tumor surgery. Frontiers in Neurology 12:711646

9. de Witt Hamer PC, Gil Robles S, Zwinderman AH, Duffau H, Berger MS (2012) Impact of intraoperative stimulation brain mapping on glioma surgery outcome: a meta-analysis. 30(20):2559-2565

10. Dick AS, Tremblay P (2012) Beyond the arcuate fasciculus: consensus and controversy in the connectional anatomy of language. Brain 135(12):3529-3550

11. Fornia L, Puglisi G, Leonetti A, Bello L, Berti A, Cerri G (2020) Garbarini F (2020) Direct electrical stimulation of the premotor cortex shuts down awareness of voluntary actions. Nat Commun 11(1):705

12. Geschwind $N$ (1970) The organization of language and the brain. Science 170(3961):940-944

13. Glasser MF, Coalson TS, Robinson EC et al (2016) A multi-modal parcellation of human cerebral cortex. Nature 536(7615):171-178

14. Hamann JM, Dayan E, Hummel FC, Cohen LG (2014) Baseline frontostriatal-limbic connectivity predicts reward-based memory formation. Hum Brain Mapp 35(12):5921-5931

15. Harvey PD (2019) Domains of cognition and their assessment. Dialogues Clin Neurosci 21(3):227-237

16. Herbet $\mathrm{G}$ (2021) Should complex cognitive functions be mapped with direct electrostimulation in wide-awake surgery? A network perspective. Frontiers in Neurology 12:635439

17. Isitan C, Yan Q, Spencer DD, Alkawadri R (2020) Brief history of electrical cortical stimulation: a journey in time from Volta to Penfield. Epilepsy Research 166:106363

18. Klitsinikos D, Ekert JO, Carels A, Samandouras G (2021) Mapping and anatomo-surgical techniques for SMA-cingulum-corpus callosum gliomas; how I do it. Acta Neurochir 163(5):1239-1246

19. Kong NW, Gibb WR, Tate MC (2016) Neuroplasticity: insights from patients harboring gliomas. Neural Plast 2016:2365063

20. Landers MJF, Sitskoorn MM, Rutten GJM, Mandonnet E, de Baene W (2021) A systematic review of the use of subcortical intraoperative electrical stimulation mapping for monitoring of executive deficits and neglect: what is the evidence so far? Acta Neurochirurgica

21. Leblanc R (2018) Cushing, Penfield, and cortical stimulation. J Neurosurg 130(1):76-83

22. Mahon BZ, Miozzo M, Pilcher WH (2019) Direct electrical stimulation mapping of cognitive functions in the human brain. Cogn Neuropsychol 36(3-4):97-102

23. Makris N, Kennedy DN, McInerney S, Sorensen AG, Wang R, Caviness VS Jr, Pandya DN (2005) Segmentation of subcomponents within the superior longitudinal fascicle in humans: a quantitative, in vivo. DT-MRI study Cerebral cortex 15(6):854-869

24. Nieuwenhuys R (2013) The myeloarchitectonic studies on the human cerebral cortex of the Vogt-Vogt school, and their significance for the interpretation of functional neuroimaging data. Brain Struct Funct 218(2):303-352

25. Niki C, Kumada T, Maruyama T, Tamura M, Kawamata T, Muragaki Y (2020) Primary cognitive factors impaired after glioma surgery and associated brain regions. Behav Neurol 2020:7941689 
26. Ojemann GA (1983) Brain organization for language from the perspective of electrical stimulation mapping. Behavioral and Brain Sciences 6(2):189-206

27. Papagno C (2017) Studying cognitive functions by means of direct electrical stimulation: a review. Neurol Sci 38(12):2079-2087

28. Perrone-Bertolotti M, Alexandre S, Jobb AS, de Palma L, Baciu M, Mairesse MP, Hoffmann D, Minotti L, Kahane P, David O (2020) Probabilistic mapping of language networks from high frequency activity induced by direct electrical stimulation. Hum Brain Mapp 41(14):4113-4126

29. Puglisi G, Howells H, Sciortino T et al (2019) Frontal pathways in cognitive control: direct evidence from intraoperative stimulation and diffusion tractography. Brain 142(8):2451-2465

30. Rahimpour S, Haglund MM, Friedman AH, Duffau H (2019) History of awake mapping and speech and language localization: from modules to networks. Neurosurg Focus 47(3):E4

31. Ruis C (2018) Monitoring cognition during awake brain surgery in adults: a systematic review. J Clin Exp Neuropsychol 40(10):1081-1104

32. Sarubbo S, Tate M, de Benedictis A, Merler S, Moritz-Gasser S, Herbet G, Duffau H (2020) Mapping critical cortical hubs and white matter pathways by direct electrical stimulation: an original functional atlas of the human brain. NeuroImage. 205:116237

33. Sefcikova V, Christofi G, Samandouras G (2021) Commentary: post-acute cognitive rehabilitation for adult brain tumor patients. Neurosurgery. nyab064

34. Sefcikova V, Sporrer JK, Ekert JO, Kirkman MA, Samandouras G (2020) High interrater variability in intraoperative language testing and interpretation in awake brain mapping among neurosurgeons or neuropsychologists: an emerging need for standardization. World neurosurgery 141:e651-e660

35. Shallice T, Cipolotti L (2018) The prefrontal cortex and neurological impairments of active thought. Annu Rev Psychol 69:157-180

36. Vilensky JA, Gilman S (2002) Horsley was the first to use electrical stimulation of the human cerebral cortex intraoperatively. Surg Neurol 58(6):425-426

37. Wang X, Pathak S, Stefaneanu L, Yeh FC, Li S, FernandezMiranda JC (2016) Subcomponents and connectivity of the superior longitudinal fasciculus in the human brain. Brain Struct Funct 221(4):2075-2092

38. Yagmurlu K, Vlasak AL, Rhoton AL Jr (2015) Three-dimensional topographic fiber tract anatomy of the cerebrum. Neurosurgery 11(Suppl 2):274-305

39. Yordanova YN, Duffau H, Herbet G (2017) Neural pathways subserving face-based mentalizing. Brain Struct Funct 222(7):3087-3105

40. Zhang JJY, Lee KS, Voisin MR, Hervey-Jumper SL, Berger MS, Zadeh G (2020) Awake craniotomy for resection of supratentorial glioblastoma: a systematic review and meta-analysis. Neurooncology advances 2(1):vdaa111

Publisher's note Springer Nature remains neutral with regard to jurisdictional claims in published maps and institutional affiliations. 